\title{
Change detection of bitemporal multispectral images based on FCM and D-S theory
}

\author{
Aiye Shi ${ }^{1 *}$, Guirong Gao ${ }^{1}$ and Shaohong Shen ${ }^{2}$
}

\begin{abstract}
In this paper, we propose a change detection method of bitemporal multispectral images based on the D-S theory and fuzzy c-means (FCM) algorithm. Firstly, the uncertainty and certainty regions are determined by thresholding method applied to the magnitudes of difference image (MDI) and spectral angle information (SAI) of bitemporal images. Secondly, the FCM algorithm is applied to the MDI and SAI in the uncertainty region, respectively. Then, the basic probability assignment (BPA) functions of changed and unchanged classes are obtained by the fuzzy membership values from the FCM algorithm. In addition, the optimal value of fuzzy exponent of FCM is adaptively determined by conflict degree between the MDI and SAI in uncertainty region. Finally, the D-S theory is applied to obtain the new fuzzy partition matrix for uncertainty region and further the change map is obtained. Experiments on bitemporal Landsat TM images and bitemporal SPOT images validate that the proposed method is effective.
\end{abstract}

Keywords: Multitemporal, Multispectral, Change detection, D-S theory, FCM

\section{Introduction}

Change detection is referred to observing and processing the same area of multitemporal images at different time. It can provide monitoring information of change for government and has been applied to many domains such as forestry monitoring, natural diaster monitoring, and the urban development $[1,2]$. In general, change detection technique can be divided into two main categories: unsupervised [3-14] and supervised change detection methods $[15,16]$.

Among the unsupervised change detection methods, change vectors analysis (CVA) techniques are widely used $[3,6,13]$. The technique firstly computes the difference image (DI), and the magnitudes of DI (MDI) are segmented into unchanged and changed classes. Like other unsupervised change detection methods, how to select a suitable threshold is an open problem for CVA techniques. Furthermore, even if a better threshold for a certain unsupervised change detection method is obtained,

\footnotetext{
*Correspondence: ayshi.hhu@gmail.com

${ }^{1}$ College of Computer and Information, Hohai University, No.8, Focheng West Road, Nanjing, China
}

Full list of author information is available at the end of the article the region around the threshold is still difficult to judge the pixels' class (change and unchange). This problem is partially due to the loss of information associated with the difference and magnitude operators, which do not allow to exploit all the information of the original feature space in the change detection process [4].

Another important change detection methods are transform-based methods. These methods include principle component analysis [17], multivariate alteration detection [18], and chi-squared transform methods [19, 20]. The most advantage of these methods is in reducing data redundancy between bands and emphasizing different information in derived components. However, it is difficult for interpreting and labeling the change information on the transformed images.

In the past few years, many pattern recognition algorithms, such as support vector machine [4] and deep learning neural networks [11], have been applied for the change detection of remotely sensed images. In these algorithms, fuzzy c-means (FCM) algorithms, which can get the degree of uncertainty of feature data belonging to each class and expresses the intermediate property of their memberships, have been widely used in the change 
detection [8, 10, 12, 21-24]. Gong et al. in [10] proposed a change detection method based on the combination of FCM and Markov random field (MRF). The method has a good computational performance by modifying the membership instead of modifying the objective function. In addition, the membership of each pixel are constructed by a novel form of MRF energy function. In [21], FCM and GustafsonCKessel clustering algorithms were used for change detection. At the same time, the 8-neighbor and 12-neighbor pixels as spatial information are used in the FCM. In addition, the genetic algorithm and simulated annealing were used to optimize the object function of FCM to further enhance the CD performance. In [23], the integration of FCM and MRF is applied to change detection in multispectral and multitemporal remote sensing images. In this study, MRF is used to model the spatial gray level attributes of the multispectral difference image.

The advantage of FCM algorithms need not to determine the threshold. However, there are two shortcomings for the FCM algorithm applied to the MDI. One is the loss of the original spectral information because of only the single information being used, which causes the FCM algorithm to be the worse result in the uncertainty region (around the threshold). Another problem is that the fuzzy exponent of FCM is not easily determined, which is generally acquired by try and error method or empirical knowledge. The methods make the FCM have no generality for change detection. In order to overcome the above shortcomings, we use the magnitude and spectral angle information of bitemporal image in the uncertainty region. Then, we use the D-S theory to fuse the results from the magnitude and spectral angle in order to reduce the uncertainty. This is because the D-S theory has the advantage of processing uncertainty and fusing the different information $[25,26]$. In addition, the fuzzy exponent of FCM objective function is adaptively determined by the total conflict degree between the MDI and spectral angle information (SAI) of uncertainty region in bitemporal images.

The main contributions of our wok are as follows: (1) the certainty and uncertainty regions are determined by fusing the results of MDI and SAI. (2) The fuzzy exponent of FCM objective function is adaptively determined by conflict degree of evidence between MDI and SAI. (3) $\mathrm{D}-\mathrm{S}$ theory is applied to increase the reliability of change detection in the uncertainty region.

In the following sections, we first briefly introduce the principle of D-S theory. Secondly, the FCM algorithm is introduced. Then, our proposed change detection method is described. After that, the experiments on two bitemporal remotely sensed images are conducted to evaluate our proposed method. Finally, the conclusions are given.

\section{D-S theory}

The Dempster-Shafer (D-S) theory was developed by Arthur P. Dempster [27] and generalized by Glenn Shafer [28]. The D-S theory, also known as the theory of belief functions, is a generalization of the Bayesian theory of subjective probability. Whereas the Bayesian theory requires probabilities for each question of interest, belief functions allow us to base belief degrees for one question on probabilities to a related question. These degrees of belief may or may not have the mathematical properties of probabilities. This theory is a mathematical theory of evidence [27] based on belief functions and plausible reasoning, which is used to combine separate pieces of information (evidence) to calculate the probability of an event.

In D-S theory, there is a fixed set of $Q$ mutually exclusive and exhaustive elements, called the frame of discernment, which is symbolized by:

$$
\Theta=\left\{H_{1}, H_{2}, \cdots, H_{Q}\right\}
$$

The representation scheme, $\Theta$, defines the working space for the desired application since it consists of all propositions for which the information sources can provide evidence.

Define function $m$ be the reflection from the set $2^{\Theta}$ to the range $[0,1]$ and satisfies the following:

$$
\left\{\begin{array}{l}
m(\phi)=0, \\
\sum_{A_{i} \in 2^{\Theta}} m\left(A_{i}\right)=1
\end{array}\right.
$$

$m(A)$ is defined as the basic probability assignment (BPA) function of hypothesis $A$.

The belief and plausibility functions are derived from the BPA function, and are respectively defined by

$$
\begin{aligned}
& \left\{\begin{array}{l}
\operatorname{bel}(\phi)=0, \\
\operatorname{bel}(A)=\sum_{B \subset A} m(B), \forall A \subset \Theta
\end{array}\right. \\
& \left\{\begin{array}{l}
\operatorname{pl}(\phi)=0, \\
\operatorname{pl}(A)=\sum_{B \cap A \neq \phi} m(B), \forall A \subset \Theta
\end{array}\right.
\end{aligned}
$$

BPA from different information sources, $m_{j}(j=$ $1, \cdots, d)$, are combined with Dempster's orthogonal rule. The result is a new distribution, $m\left(A_{k}\right)=(m 1 \oplus m 2 \oplus$ $\cdots \oplus m d)\left(A_{k}\right)$, which incorporates the joint information provided by the sources and can be represented as follows:

$$
\begin{aligned}
& m\left(A_{k}\right)=\frac{\sum_{A_{1} \cap A_{2} \cdots A_{d}=A_{k}} \prod_{1 \leq j \leq d} m_{j}\left(A_{j}\right)}{1-K} \\
& K=\sum_{A_{1} \cap A_{2} \cdots A_{d}=\phi}\left(\prod_{1 \leq j \leq d} m_{j}\left(A_{j}\right)\right)
\end{aligned}
$$

$K$ is often interpreted as a measure of conflict between the different sources and is introduced as a normalization factor. The larger $K$ is the more the sources are conflicting and the less sense has their combination. The factor $K$ 
indicates the amount of evidential conflict. If $K=0$, this shows complete compatibility, and if $0<K<1$, it shows partial compatibility. Finally, the orthogonal sum does not exist when $K=1$. In this case, the sources are totally contradictory, and it is no longer possible to combine them. In the cases of sources highly conflicting, the normalization used in the Dempster combination rule can be mistaking, since it artificially increases the masses of the compromise hypotheses.

\section{FCM}

Fuzzy c-means was firstly proposed by Dunn [29] and generalized by Bezdek [30]. The FCM algorithm classifies images by grouping points with similar features into clusters. FCM algorithm is the improvement of K-means algorithm. In change detection problem, FCM algorithm is a soft partition for changed and unchanged class. The idea of FCM is that make the object in the same cluster have the largest similarity and least similarity between different clusters. The algorithm iteratively minimizes a objective function which depends on the pixels to the cluster centers in the feature domain.

Let a dataset $\left\{\mathbf{x}_{k}\right\}_{k=1}^{N} \in R^{d}$ to be partitioned into $c$ clusters, then the definition of objective function is as follows:

$$
J_{q}=\sum_{i=1}^{c} \sum_{k=1}^{N}[u(i, k)]^{q}\left\|\mathbf{x}_{k}-\mathbf{v}_{i}\right\|^{2}
$$

where the element $u(i, k)$ of fuzzy partition matrix is the membership of the $k$ th sample corresponding to the center $v_{i}$ of $i$ th class, $u(i, k) \in[0,1]$ and $\sum_{i=1}^{c} u(i, k)=1, q$ is the weighted exponent on each fuzzy membership and $q \in(1, \infty)$.

The objective function in (6) is minimized using the following alternate iterations:

$$
\begin{aligned}
& u(i, k)=\frac{1}{\sum_{j=1}^{c}\left(\frac{\left\|\mathbf{x}_{k}-\mathbf{v}_{i}\right\|}{\left\|\mathbf{x}_{k}-\mathbf{v}_{j}\right\|}\right)^{\frac{2}{(q-1)}}} \\
& \mathbf{v}_{i}=\frac{\sum_{k=1}^{N}[u(i, k)]^{q} \mathbf{x}_{k}}{\sum_{k=1}^{N}[u(i, k)]^{q}}
\end{aligned}
$$

\section{Change detection based on FCM algorithm and D-S theory}

Let $X_{1}$ and $X_{2} \in \mathbb{R}^{H_{1} \times H_{2} \times B}$ be two temporal images consisting of $B$ bands, where $H_{1}$ and $H_{2}$ are the height and the width of the image, respectively. We assume that both images have been co-registered and radiometrically corrected.

The proposed method includes three main parts (as shown in Fig. 1): (1) the uncertainty and certainty regions are determined by combining the threshold of MDI with the one of SAI; (2) construction of mass function based on FCM algorithm and then D-S evidence combination for

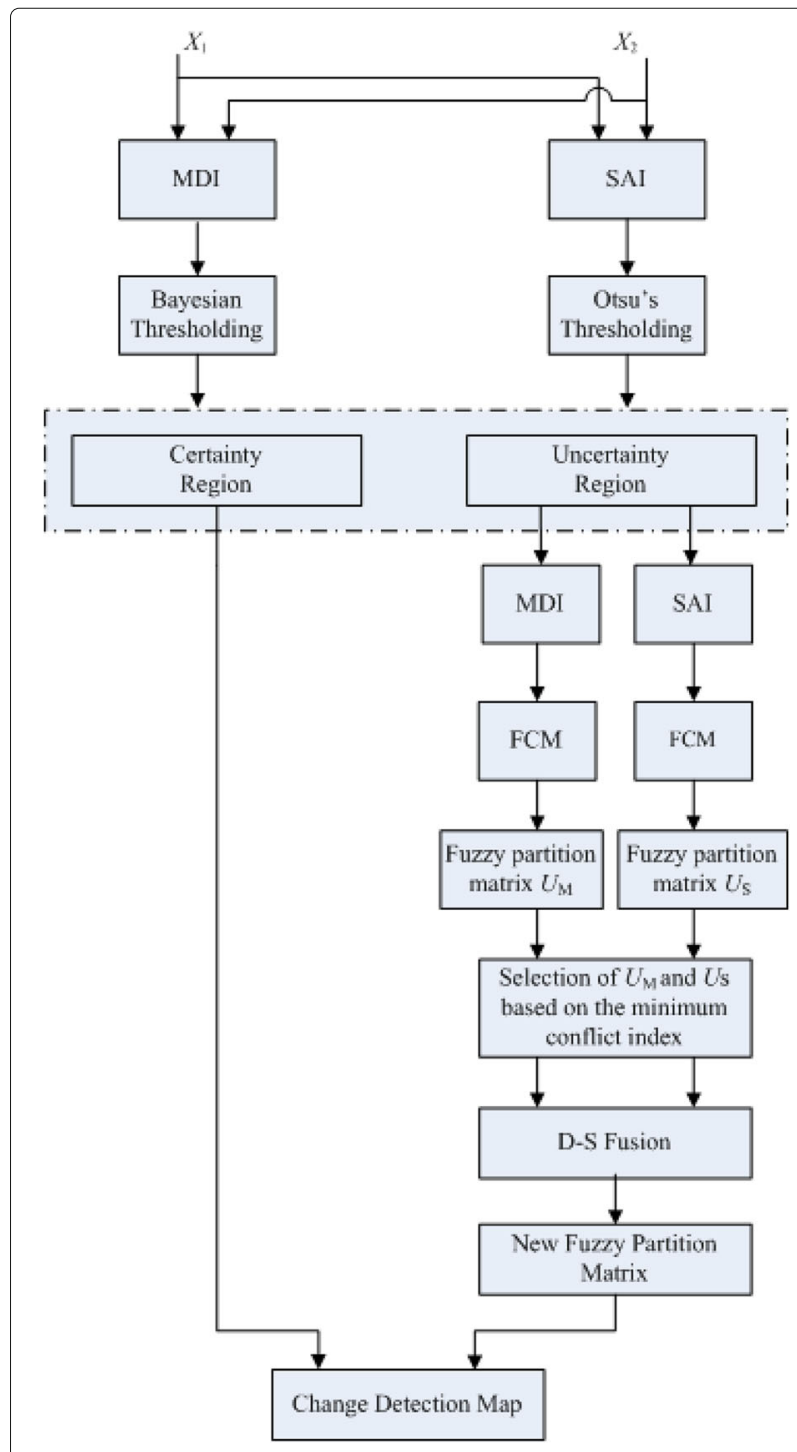

Fig. 1 The diagram of proposed change detection method

the MDI and SAI in uncertainty regions; and (3) parameter optimization based on conflict index. The following sections give the description of these three main parts.

4.1 The determination of uncertainty and certainty region Let $\mathbf{M}$ and $\mathbf{S}$ represent the MDI and SAI of $\mathbf{X}_{1}$ and $\mathbf{X}_{2}$, respectively. The pixel values at location $(i, j)$ of $\mathrm{MDI}$ and SAI are denoted by $M(i, j)$ and $S(i, j)$, respectively, and are expressed as follows:

$$
M(i, j)=\sqrt{\sum_{b=1}^{B}\left(X_{1 b}(i, j)-X_{2 b}(i, j)\right)^{2}} .
$$




$$
S(i, j)=\arccos \left(\frac{\sum_{b=1}^{B}\left(X_{1 b}(i, j) X_{2 b}(i, j)\right)}{\sqrt{\sum_{b=1}^{B} X_{1 b}^{2}(i, j)} \sqrt{\sum_{b=1}^{B} X_{2 b}^{2}(i, j)}}\right)
$$

where $X_{1 b}(i, j)$ and $X_{2 b}(i, j)$ represent the value of the $b$ th band of images $\mathbf{X}_{1}$ and $\mathbf{X}_{2}$ at location $(i, j)$, respectively.

We reformulate $\mathbf{M}$ and $\mathbf{S}$ as a column vector by lexicographically ordering the pixels on the image and denote the two matrices by $\widetilde{\mathbf{M}}$ and $\widetilde{\mathbf{S}}$, respectively. The values of $\widetilde{\mathbf{M}}(p)$ and $\widetilde{\mathbf{S}}(p)$ are the $p$ th element of column vector of MDI and SAI, respectively.

In this work, we only cope with abrupt change detection; therefore, there are two classes: unchanged and changed classes. Based on Bayes rule, we adopt expectation maximization (EM) algorithm to find the threshold $T_{M}$ of MDI. In general, a magnitude value that is close to the threshold, the much uncertainty it is.

The threshold value $T_{M}$ represents a reasonable reference point for identifying uncertainty and certainty regions. According to this observation, the desired set of pixels with a high probability to be correctly assigned to one of the two classes, i.e., certainty regions, is constructed as follows [4, 31]:

(1) The region where the values of MDI are less than $T_{M}-\delta_{1}$ is considered unchanged class.

(2) The region where the values of MDI are larger than $T_{M}+\delta_{2}$ is considered changed class.

In the definition, $\delta_{1}$ and $\delta_{2}$ are both positive constants, whose values should be selected in order to obtain a high probability that patterns in MDI have a correct label. It is worth noting that, in general, the margin can be approximated as symmetric with respect to the threshold; thus, we can assume $\delta_{1}=\delta_{2}=\delta$. A reasonable strategy for selecting the value of $\delta$ is to relate it to the dynamic range of the difference image. The choice of $\delta$ should make the value of $T_{M}-\delta$ be greater than zero. Generally, $\delta$ is chosen to be less than $15 \%$ of dynamic range of MDI. In [31], the authors chose the $\delta$ to be a constant value. Shao et al. in [24] chosen the parameters $T_{M}-\delta_{1}$ and $T_{M}+\delta_{2}$ to be the mean of unchanged region and changed region based on the threshold $T_{M}$, respectively.

Although we can choose uncertainty and certainty regions based on the method in $[4,24,31]$, the above methods only use the MDI information and this information cannot be enough to reflect the change and unchange information, which will lead to some labels to be misclassified in certainty region. In order to further decrease misclassified pixels in the certainty regions based on Bayes rule with change vectors, we use another feature, spectral angle information, to refine the certainty and uncertainty regions set obtained from MDI.
In this work, we apply Otsu's thresholding method to determine the threshold of spectral angle [32]. The SAI includes two types of classes: changed and unchange pixels. The Otsu's algorithm then calculates the optimum threshold separating the two classes so that their combined spread (intra-class variance) is minimal, or equivalently (because the sum of pairwise squared distances is constant), so that their inter-class variance is maximal.

Suppose the threshold of SAI by Otsu's method be $T_{S}$. Let certainty region $\mathcal{P}_{l}$ includes two subsets: unchanged region $\mathcal{P}_{u}$ and changed region $\mathcal{P}_{c}$. That is $\mathcal{P}_{l}=\mathcal{P}_{u} \cup \mathcal{P}_{c}$. Then, we refine the certainty region as follows:

$$
\begin{aligned}
& \mathcal{P}_{u}=\left\{p \mid \tilde{M}(p) \leq T_{M}-\delta \quad \text { and } \quad \tilde{S}(p) \leq T_{S}\right\}_{p=1}^{N} \\
& \mathcal{P}_{c}=\left\{p \mid \tilde{M}(p) \geq T_{M}+\delta \quad \text { and } \quad \tilde{S}(k) \geq T_{S}\right\}_{p=1}^{N}
\end{aligned}
$$

According to the properties of MDI and SAI, the pseudolabels of pixels in $\mathcal{X}$ are assigned as follows:

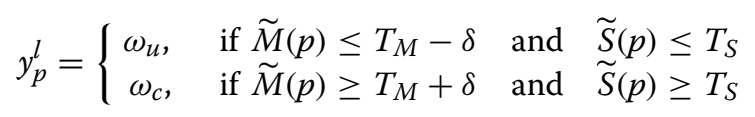

Based on Eqs. (11) and (12), the uncertainty region is defined as $\mathcal{P}_{l}^{c}=\left\{1,2, \cdots, H_{1} \times H_{2}\right\}-\mathcal{P}_{l}$. Concretely, the entire uncertainty region includes three parts (as shown in (Fig. 2)): uncertainty regions $1-3$. Uncertainty region 1 includes the locations where the values of MDI are between $T_{M}-\delta$ and $T_{M}+\delta$. Uncertainty region 2 includes the locations where the values of MDI are smaller than $T_{M}-\delta 1$ and the values of SAI are greater than $T_{S}$. Uncertainty region 3 includes the locations where the values of MDI are greater than $T_{M}+\delta$ and the values of SAI are smaller than $T_{S}$. The labels of pixels belong to the uncertain set $\mathcal{P}_{l}^{c}$ are obtained by the D-S theory and FCM algorithm.

\subsection{Construction of mass function based on FCM and D-S evidence combination}

When the FCM algorithm is applied to the MDI and SAI of uncertainty region, we obtain the fuzzy partition matrix $U_{M}$ and $U_{S}$, respectively. Because the value of partition matrix represents the membership of a sample belonging to a class, we can directly use the membership value of partition matrix as the BPA or mass function of D-S theory.

In change detection problem, the frame of discernment $\Theta=\{u, c\}$, where $u$ represents unchanged class and $c$ represents changed class. In our work, we consider the simple hypotheses and double hypotheses [33]. 


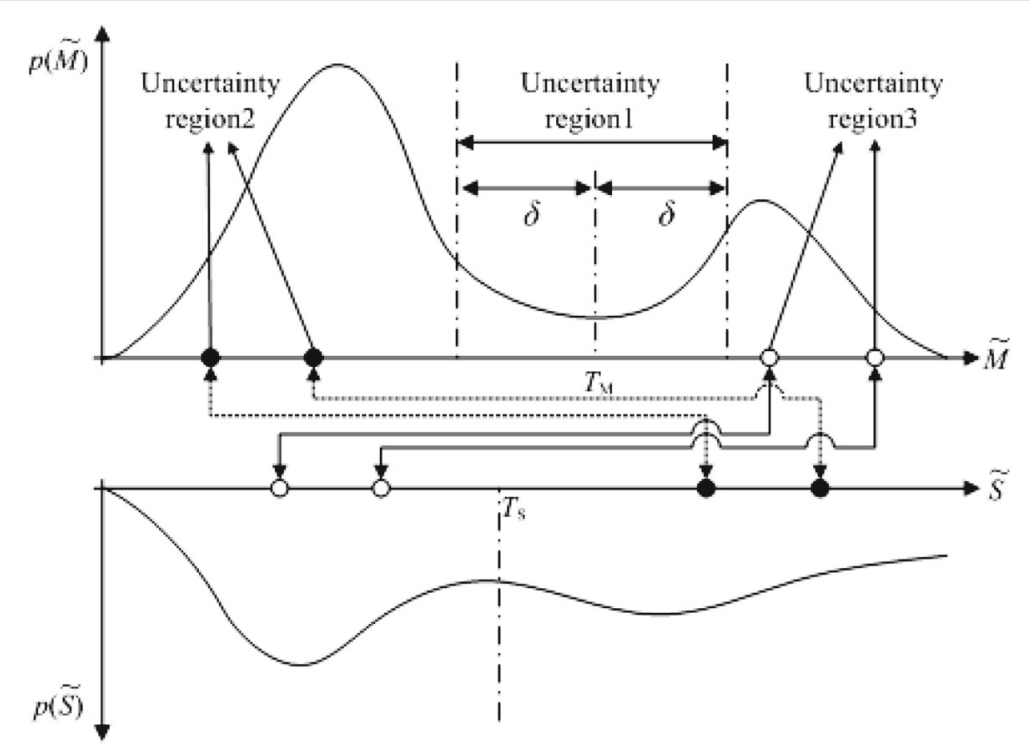

Fig. 2 Example of distributions of MDI and SAI and the definition of uncertainty region. The $P(\tilde{M})$ and $P(\tilde{S})$ represent the frequency of value of MDI and SAI, respectively

For simple hypotheses, the mass function for the $k$ th element of MDI and SAI in uncertainty region be $m_{1}^{k}$.

$$
\begin{aligned}
& m_{1}^{k}\left(A_{i}\right)=u_{M}(i, k) \\
& m_{2}^{k}\left(A_{i}\right)=u_{S}(i, k)
\end{aligned}
$$

where $i=u, c$ corresponds unchanged and changed classes.

For double hypotheses, there is a high ambiguity in assigning a pixel to unchanged class or changed class. In this case, the certain pixel's absolute of difference fuzzy membership is a smaller thresholding value (The threshold is set to be 0.1 in our work). The mass function for MDI and SAI can be represented as:

$$
\begin{aligned}
& m_{1}^{k}\left(A_{u} \cup A_{c}\right)=u_{M}(u, k) \times u_{M}(c, k) \\
& m_{2}^{k}\left(A_{u} \cup A_{c}\right)=u_{S}(u, k) \times u_{S}(c, k)
\end{aligned}
$$

After the mass functions for MDI and SAI are obtained by Eqs. (14-17), the combination rule is used by Eq. (4). When the D-S evidence combination is finished, the type of final decision output belongs to the one with the highest evidence value,

$$
F(k)=\left\{\begin{array}{cc}
\omega_{u}, & m\left(A_{c}(k)\right)<m\left(A_{u} k\right) \\
\omega_{c}, & \text { otherwise }
\end{array}\right.
$$

\subsection{Parameter optimization based on conflict index}

In the FCM objection function, the fuzzy exponent is not easily determined. In general, suitable fuzzy exponent can resist noise and balance fuzzy membership of fuzzy partition matrix. But how to select a suitable fuzzy exponent parameter is still an open problem. At present, the parameter is mainly selected by try and error method or empirical knowledge.

In this work, the appropriate fuzzy exponent $q_{1}$ for MDI and $q_{2}$ for SAI of FCM can be chosen based on grid search method. During the choice of the parameter, we abide on the following rule: the better the values of $q_{1}$ and $q_{2}$ are, the less the sum of conflict between the MDI and SAI on uncertainty region is.

Define the conflict index of uncertainty region as conflict index $(\mathrm{CI})$, which is represented as follows:

$$
\mathrm{CI}=\frac{n_{1}+n_{2}}{N_{u}}
$$

where $N_{u}$ is the total number of pixels in uncertainty region, and $n_{1}$ and $n_{2}$ are defined in uncertainty region as follows:

$n_{1}=\left\{N(k) \mid u_{M}(1, k) \geq u_{M}(2, k)\right.$ and $\left.u_{S}(1, k)<u_{S}(2, k)\right\}$

$n_{2}=\left\{N(k) \mid u_{M}(1, k) \leq u_{M}(2, k)\right.$ and $\left.u_{S}(1, k)>u_{S}(2, k)\right\}$

where $N(k)$ represents the number of pixels whose fuzzy membership for MDI and SAI are conflict in the uncertainty region. 
When the range of $q_{1}$ and $q_{2}$ are set and their steps are also set, the grid search is applied to find the suitable $q_{1}$ and $q_{2}$ according to the minimum value of $C I$ based on Eq. (19).

\section{The implementation of proposed method}

The implementation steps of proposed change detection method are as follows:

Step 1: Compute the MDI and SAI of bitemporal images, respectively.

Step 2: Determine the threshold $T_{M}$ of MDI and $T_{S}$ of SAI based on Bayesian thresholding and Otsu's threshoding methods, respectively.

Step 3: Determine the certainty region $\mathcal{P}_{l}$ according to Eqs. (11) and (12), the labels of certainty region according to Eq. (13) and further determine the uncertainty region to be $\mathcal{P}_{l}^{c}$.

Step 4: Set the grid search range of fuzzy exponent $q_{1}$ and $q_{2}$ of FCM algorithm and their increasing steps

$\Delta q_{1}$ and $\Delta q_{2}$ for the MDI and SAI of bitemporal images.

Step 5: Select the initial center of unchanged and changed classes based on certainty regions. That is, the means of MDI and SAI in certainty region are computed in advance based on Eq. (11) and taken as the initial center of unchanged class. Similarly, the means of MDI and SAI based on Eq. (12) are used to be the initial center of changed class.

Step 6: For $q_{1}^{\text {new }}=q_{1}^{\text {old }}+\Delta q_{1}$ and $q_{2}^{\text {new }}=q_{2}^{\text {old }}+\Delta q_{2}$, apply FCM algorithm to MDI and SAI of uncertainty region based on Eqs. (7) and (8) until the predefined convergency criterion or maximum iteration number is reached and then store the partition matrix.

Step 7: Compute the conflict index according to Eq. (19) and then store it.

Step 8: Repeat steps 6 and 7 until the fuzzy exponent $q_{1}$ and $q_{2}$ are all reached to the corresponding maximum value.

Step 9: Find the minimum value of change index. Step 10: Output the partition matrix of MDI and SAI corresponding to the minimum value of change index. Step 11: Apply D-S theory to fuse the partition matrix of MDI and SAI to obtain the new partition matrix based on Eqs. (4), (5), and (14)-(18).

Step 12: Obtain the labels of uncertainty region according to the new partition matrix of Step 11. Step 13: Output change detection results based on the results of Steps 3 and 12.

\section{Experiments}

To evaluate the performance of the proposed method, two remotely sensed datasets were used. Both bitemporal multispectral images have been co-registered and

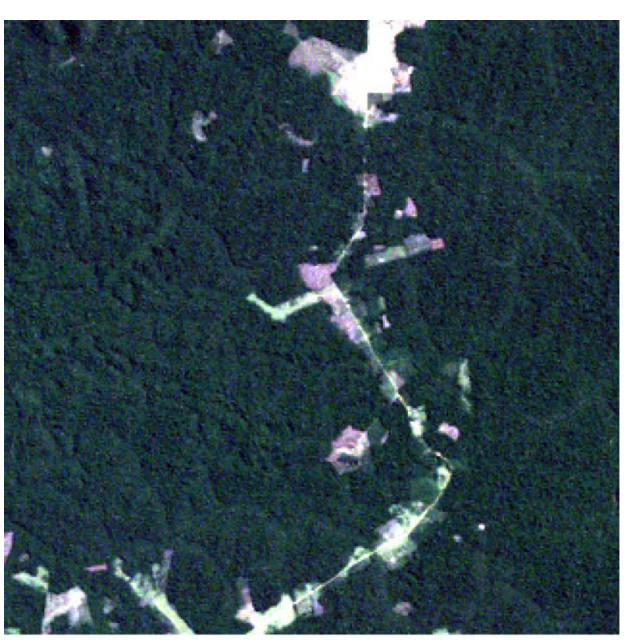

(a) The 'Before' image

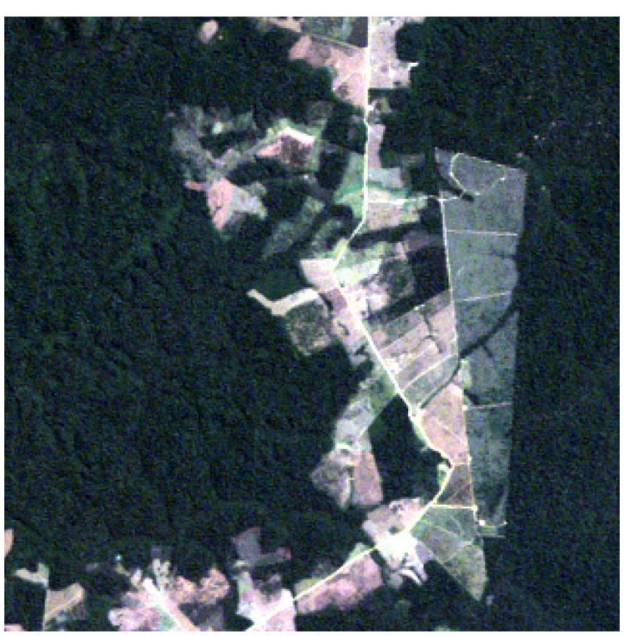

(b) The 'After' image

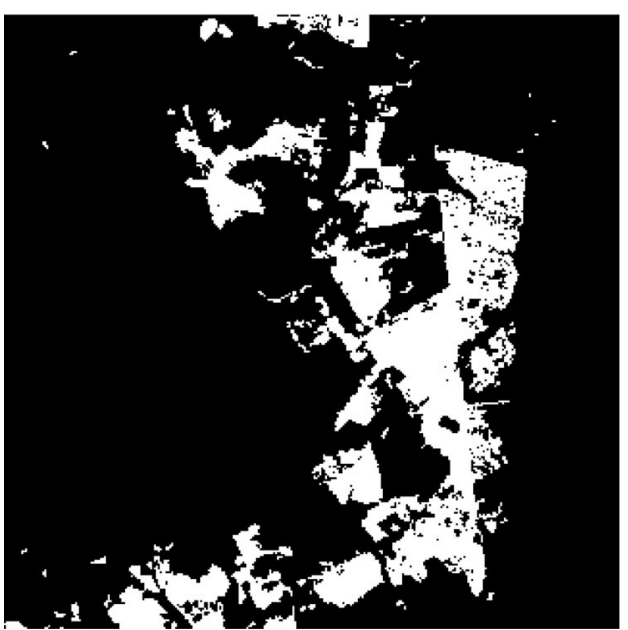

(c) The ground truth

Fig. 3 The true color images of bitemporal Brazil Landsat TM images and the ground truth 


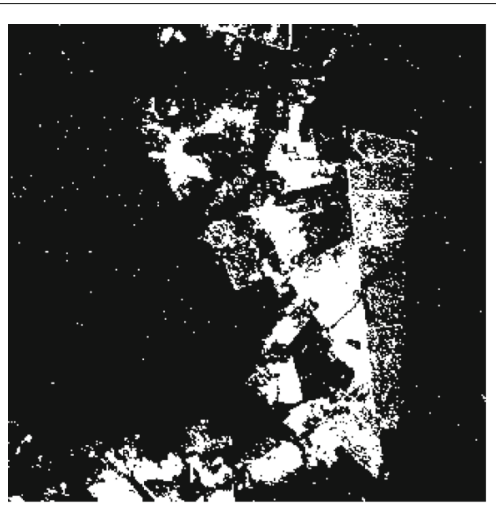

(a) EM-CVA

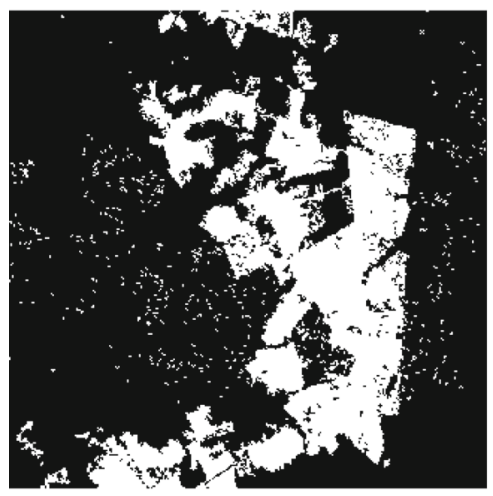

(c) FCMMRF

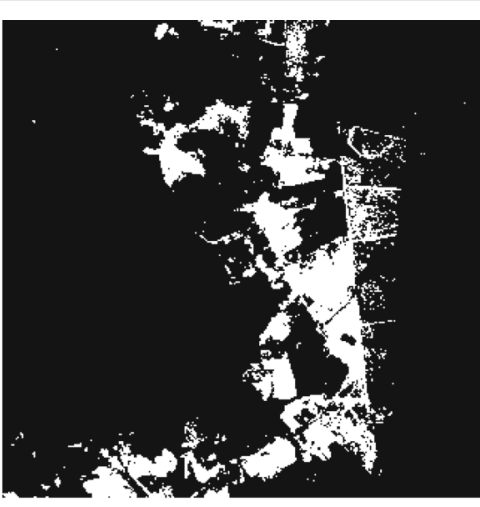

(b) RCST

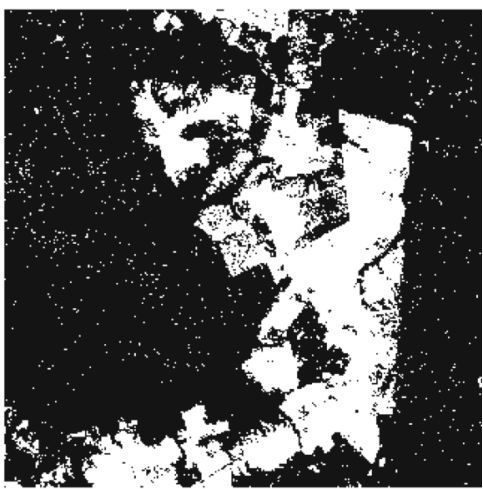

(d) HFV

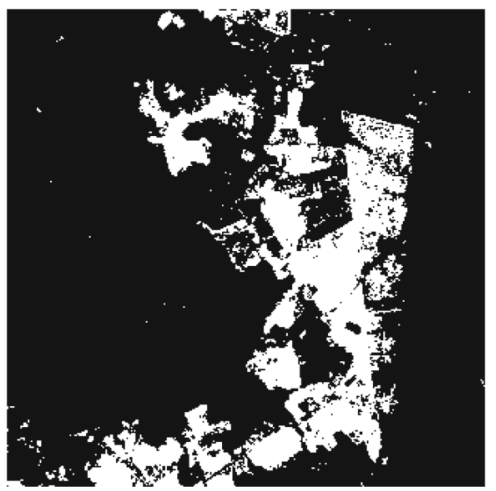

(e) Proposed

Fig. 4 The results of change detection for Brazil dataset

radiometrically corrected beforehand. The change detection results from the proposed method were compared with those from four unsupervised change detection methods, namely the EM-CVA method [3], the robust chisquared transform (RCST) method [20], the FCM algorithm combined with Markov random field (FCMMRF) on the MDI [10], and the combination of MDI and SAI (hybrid feature vector, HFV) applied with KittlerIllingworth threshold [14]. In the proposed method, the iteration number of optimization is set to 50 , the
Table 1 Change detection performance for Brazil dataset

\begin{tabular}{lllll}
\hline & FP & FN & OE & $k$ \\
\hline EM-CVA & 2918 & 3865 & 6783 & 0.753 \\
RCST & 2739 & 1864 & 4603 & 0.840 \\
FCMMRF & 8690 & 593 & 9283 & 0.723 \\
HFV & 10,299 & 20 & 10,319 & 0.705 \\
Proposed & 2537 & 870 & 3407 & 0.883 \\
\hline
\end{tabular}


convergency criterion is set to $\left\|\mathbf{V}_{\text {new }}-\mathbf{V}_{\text {old }}\right\|<0.0001$ and the value of $\delta$ is 0.1 . The fuzzy exponent is between 1.5 and 2.5, and both the values of $\Delta q_{1}$ and $\Delta q_{2}$ are set to be 0.1 .

We adopt the following four measures to assess the results: the number of false positives (FP, unchanged pixels

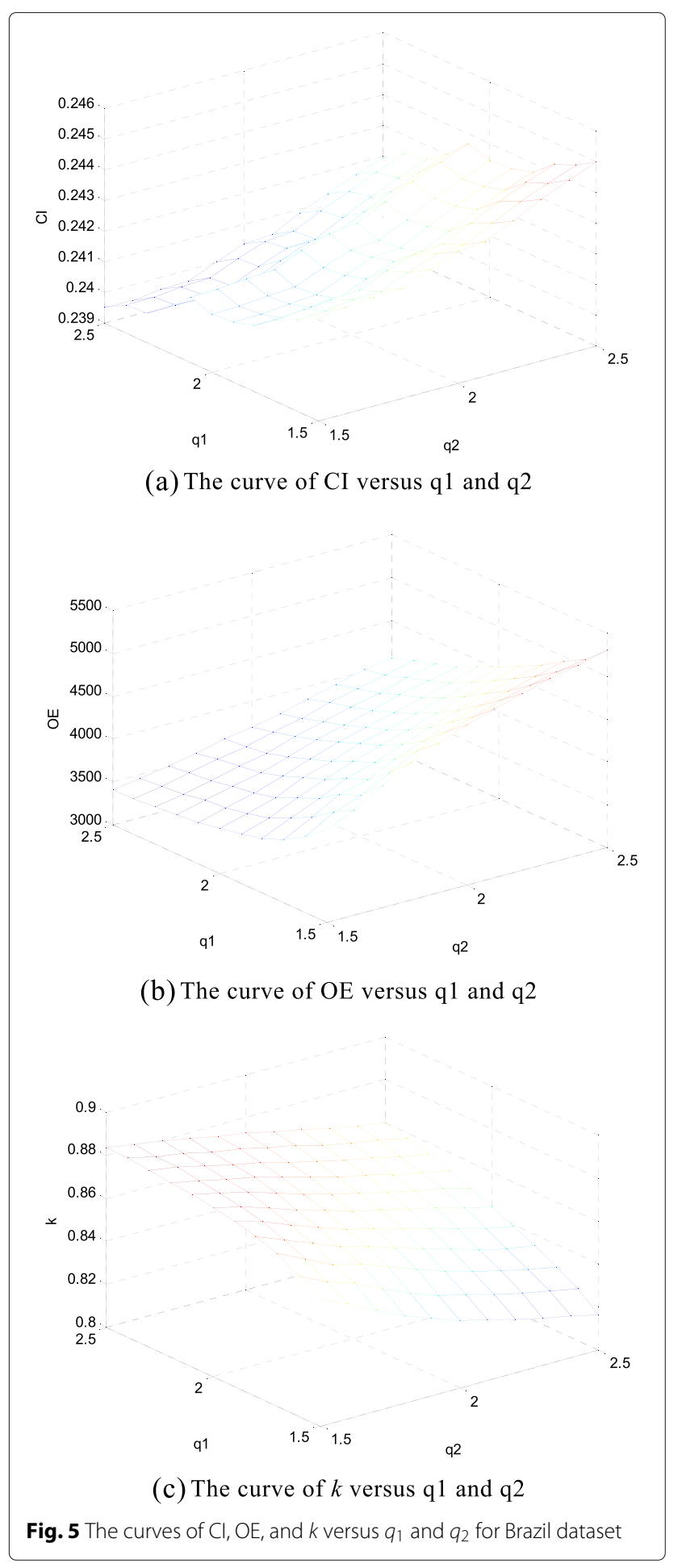

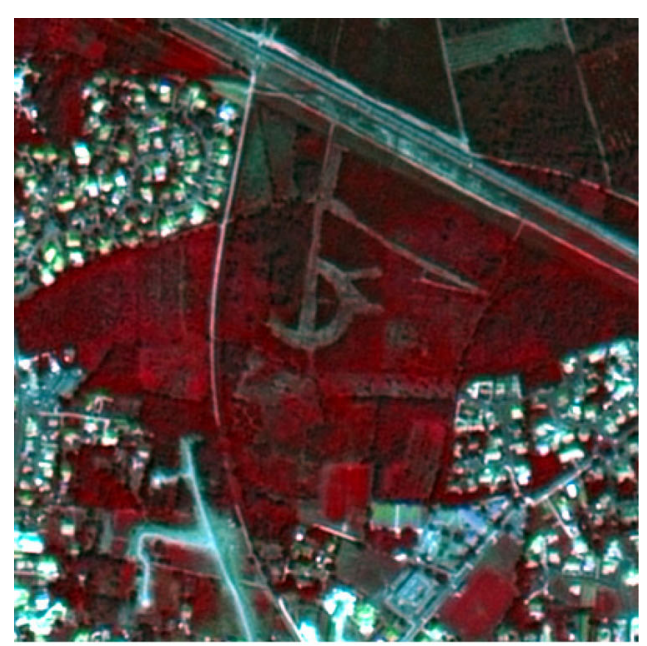

(a) The 'Before' image

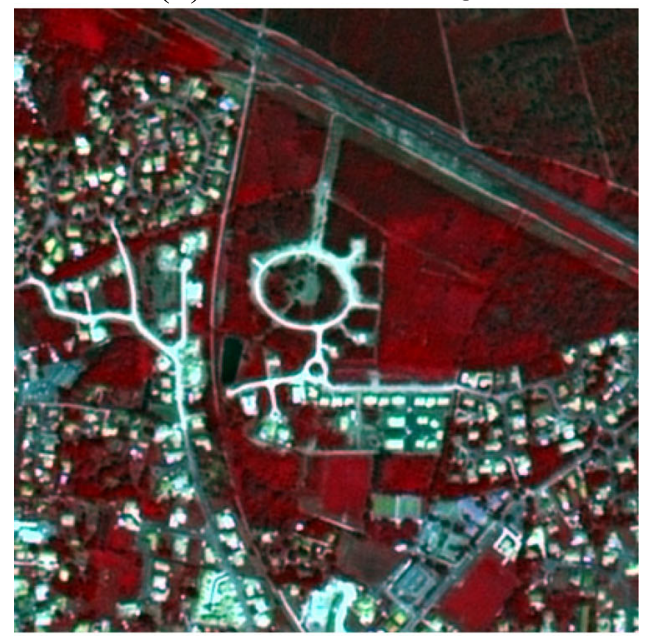

(b) The 'After' image

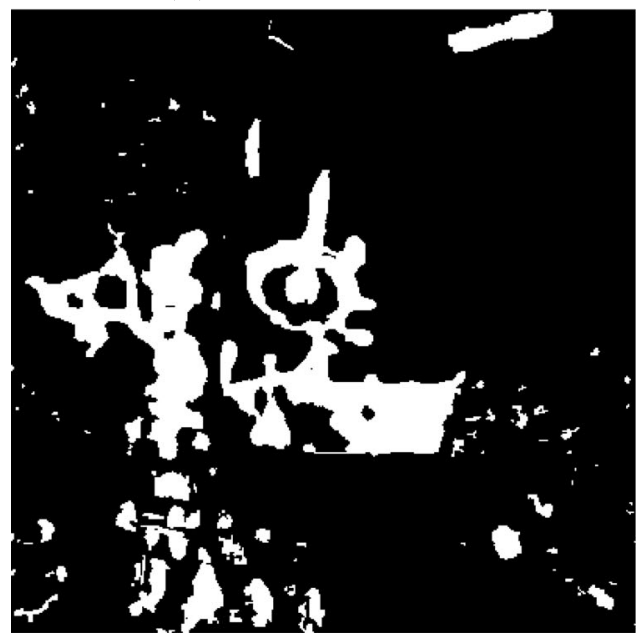

(c) The ground truth

Fig. 6 The true color images of bitemporal Littoral SPOT images and the ground truth 
wrongly classified as changed), the number of false negatives (FN, changed pixels that undetected), the overall error $(\mathrm{OE})$ defined as FP + FN, and the kappa coefficient $(\kappa)$.

\subsection{Experiments on Landsat TM imagery}

The first experiment was carried out on a section of 320 pixels $\times 320$ pixels of two multispectral images acquired by a Landsat Thematic Mapper (TM) on a forest in

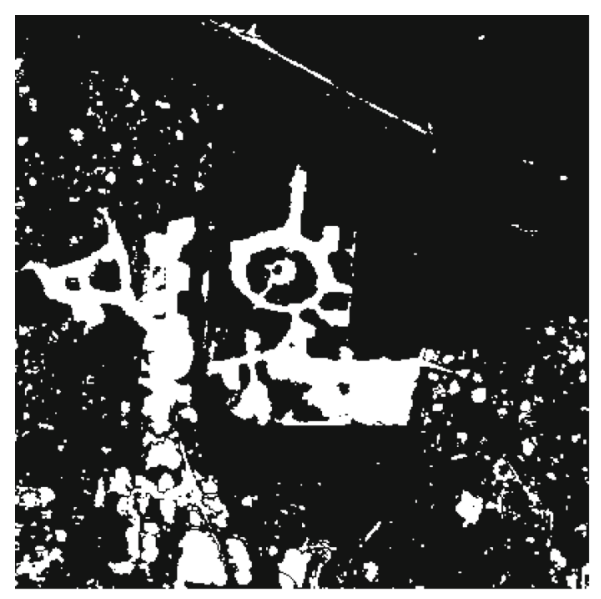

(a) EM-CVA

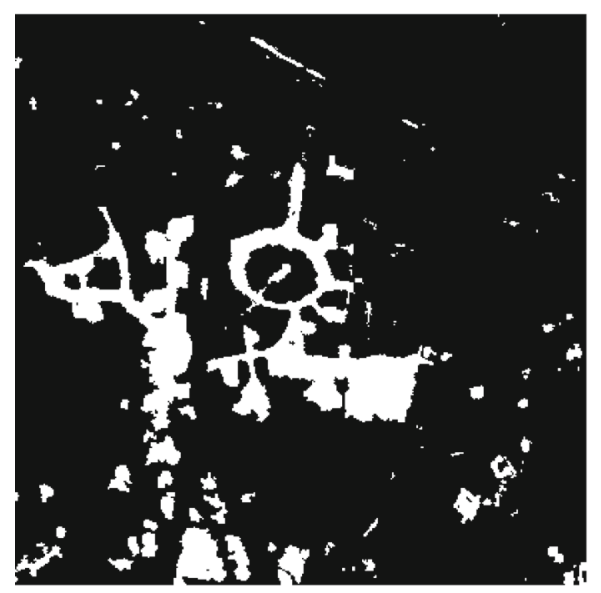

(c) FCMMRF

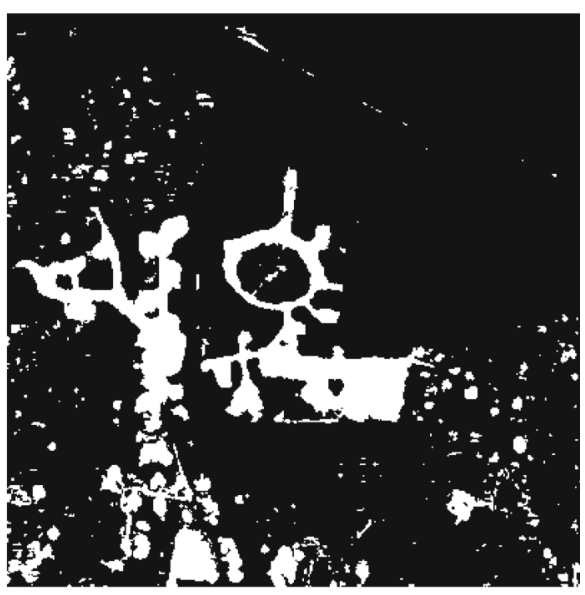

(b) RCST

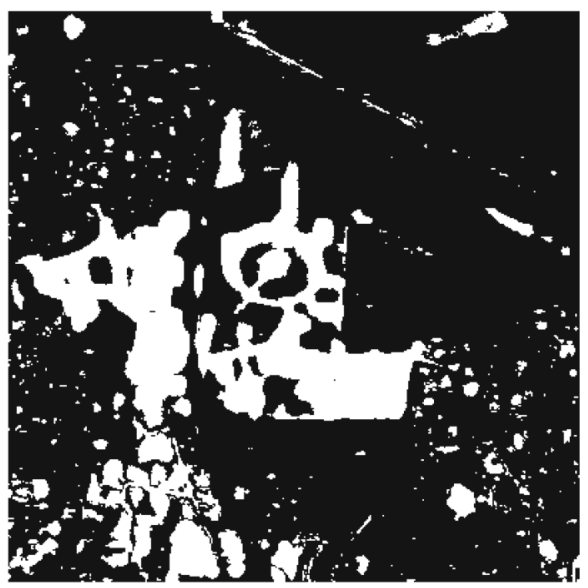

(d) HFV

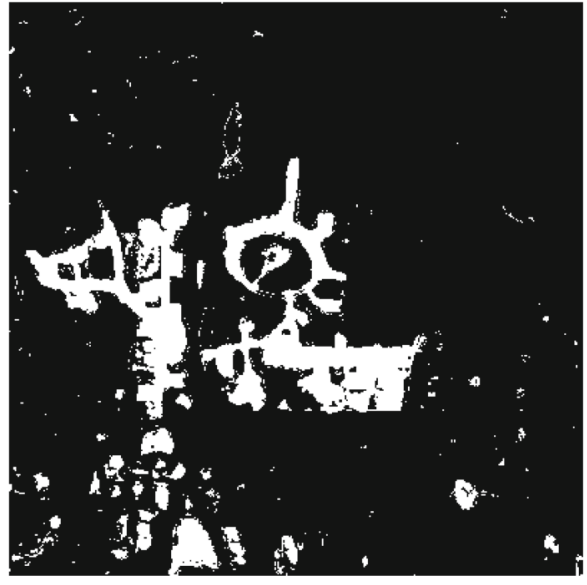

(e) Proposed

Fig. 7 The results of change detection for Littoral dataset 
Brazil. The spatial resolution of TM imagery is $30 \mathrm{~m}$. The acquisition dates of the bitemporal images were July 2000 (the "before" image) and July 2006 (the "after" image) (Fig. 3a, b), respectively. Because the visible and near infrared (NIR) bands of TM imagery contain more information about forest clearing and are useful for change detection, the four sensor bands used in the experiment were three visible bands and a NIR band.

The reference map concerning the location of the forest clearing was created manually (Fig. 3c). This ground truth map includes 16,826 changed pixels. Figure 4a-e shows the change detection results from the EM-CVA, RCST, FCMMRF, HFV, and proposed methods.

From the perspective of Fig. 4e, the change map of proposed method is closer than other methods to the ground truth data.

Table 1 presents the FP, FN, OE, and $\kappa$ values from the four state-of-the-art methods and the proposed method. The proposed method gave the best results with a change detection error of 3407 pixels. Although the FN values of our proposed method are higher than that of FCMMRF and HFV methods, our proposed method has the lowest FP values compared to other four state-of-the-art methods. In addition, our method has the lowest OE values in all the compared methods. Furthermore, we can also see from the last column that our proposed method has highest $k$ value, concretely, higher $0.13,0.04,0.16$, and 0.18 than EM-CVA, RCST, FCMMRF, and HFV methods, respectively. The comparisons show that the proposed method has the best comprehensive performance than other state-of-the-art methods.

For the effect of fuzzy exponent on the change detection, Fig. $5 \mathrm{a}-\mathrm{c}$ gives the curves of $\mathrm{CI}, \mathrm{OE}$, and $k$ versus $q_{1}$ and $q_{2}$. It can be seen that the parameters $q_{1}$ and $q_{2}$ corresponding to the minimum of CI can also obtain the highest $\mathrm{OE}$ and $k$. This shows that the parameters optimization based on the conflict index (CI) is effective.

\subsection{Experiments on SPOT imagery}

The second dataset consists of a 400 pixels $\times 400$ pixels section of two multispectral images of Kalideos Littoral acquired by a SPOT sensor from CNES in July 2006 ("before") and July 2009 ("after") (Fig. 6). The multispectral images were pansharpened by the

Table 2 Change detection performance for Littoral dataset

\begin{tabular}{lllll}
\hline Methods & FP & FN & OE & $k$ \\
\hline EM-CVA & 7918 & 3883 & 11,801 & 0.705 \\
RCST & 5020 & 5822 & 10,842 & 0.702 \\
FCMMRF & 3419 & 6057 & 9476 & 0.730 \\
HFV & 11,103 & 1427 & 12,530 & 0.716 \\
Proposed & 2255 & 6558 & 8813 & 0.739 \\
\hline
\end{tabular}

Gram-Schmidt spectral sharpening algorithm. The spatial resolution of final images is $2.5 \mathrm{~m}$. The visible bands were used in the experiments because these bands contain useful information about the variations of vegetation.

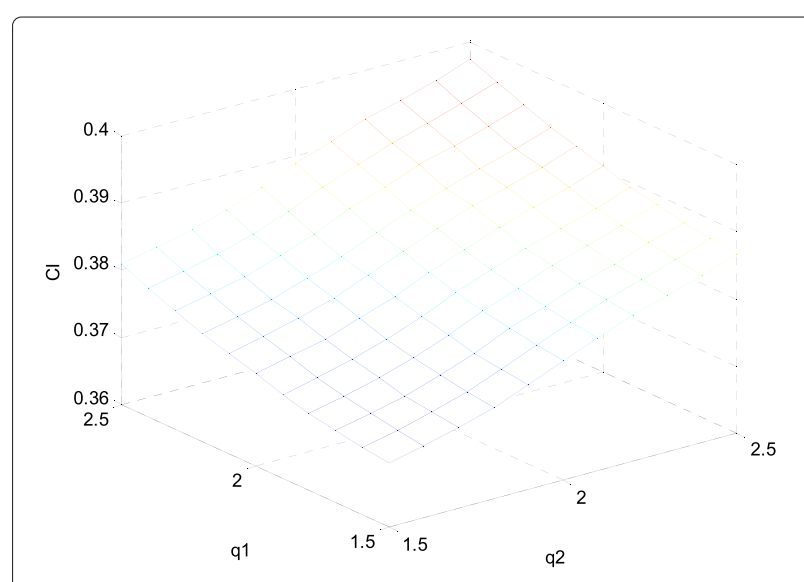

(a) The curve of CI versus $q 1$ and q2

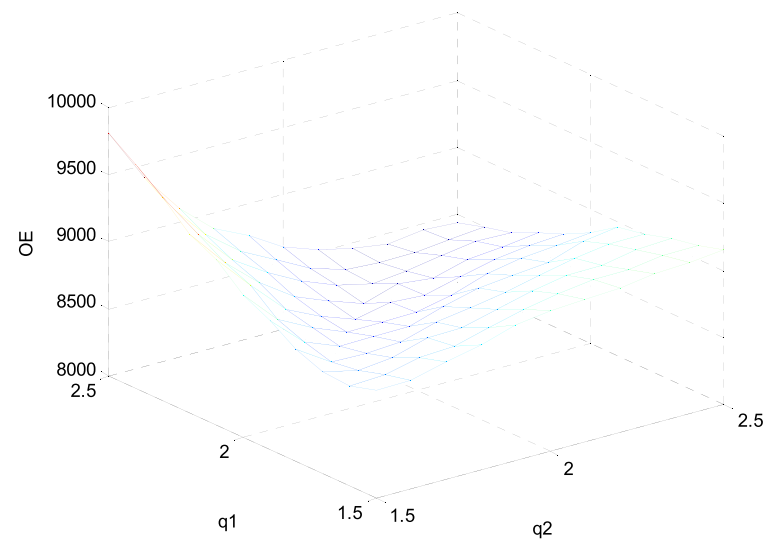

(b) The curve of OE versus q1 and q2

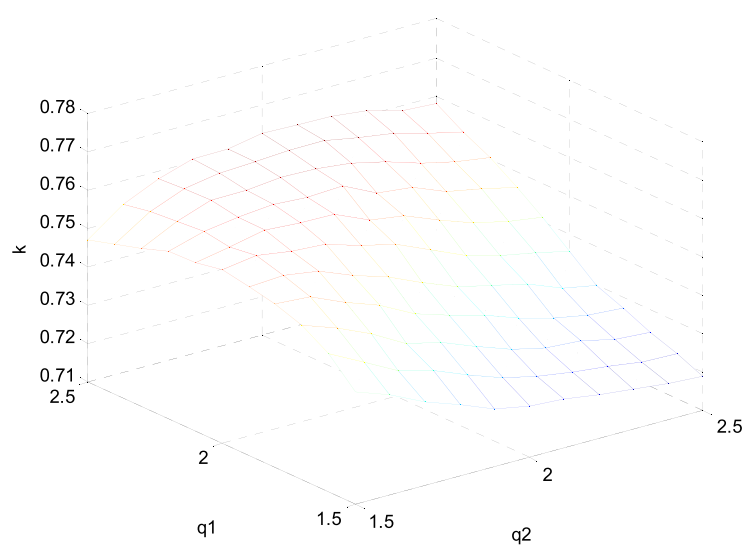

(c) The curve of $k$ versus q1 and q2

Fig. 8 The curves of $\mathrm{Cl}, \mathrm{OE}$, and $k$ versus $q_{1}$ and $q_{2}$ for Littoral dataset 
The ground reference shown in Fig. 6c was obtained from manual analysis of the two temporal images. The reference map includes 21,338 changed pixels. Figure $7 \mathrm{a}-\mathrm{e}$ shows the change detection results from the four stateof-the-art and our methods. Table 2 presents the FP, FN, $\mathrm{OE}$, and $\kappa$ values from the four state-of-the-art and the proposed methods.

The best results of our method has a change detection error of 8813 pixels, with 2255 FP and 6558 FN values. Compared to EM-CVA, RCST, and FCMMRF and HFV methods, the proposed method gives the lowest FP values but the highest FN values. In addition, the proposed method produces the lowest overall errors and the largest $\kappa$ value. From these experimental results, we can conclude that proposed method outperforms all the state-of-theart methods and is effective in change detection for this dataset.

For the effect of fuzzy exponent on the change detection, Fig. $8 \mathrm{a}-\mathrm{c}$ gives the curves of $\mathrm{CI}, \mathrm{OE}$, and $k$ versus $q_{1}$ and $q_{2}$. It can be seen that the parameters $q_{1}$ and $q_{2}$ corresponding to the minimum of CI cannot obtain the highest $\mathrm{OE}$ and $k$. From Fig. $8 \mathrm{~b}$, c, we can see that the lowest OE value and largest $k$ value are 8441 and 0.7624 , respectively. Compared to the result of proposed, we can see that the difference of $\mathrm{OE}$ and $k$ with the best value is 372 and 0.02. The performance of the proposed method is closer to the best value. This shows that proposed method is effective.

\section{Conclusions}

An unsupervised change detection method was proposed based on FCM algorithm and D-S theory, which has two features: (1) the magnitude of change vector analysis of bitemporal multispectral images with their spectral angle mapper information is fused for improving the precision of change detection based on D-S theory and (2) the fuzzy exponent parameter of FCM algorithm is adaptively determined based on the grid search. Experiments on the Brazil and Littoral datasets show that our proposed method outperforms four state-of-the-art methods.

\section{Acknowledgements}

The authors would like to thank the financial support from the Open Foundation of Changjiang Science Institute (No. CKWV2013215/KY), China, a Project Funded by the Priority Academic Program Development of Jiangsu Higher Education Institutions and the National Natural Science Foundation of China (No. 61271386). The authors thank Prof. Maoguo Gong for providing the source code of the algorithm proposed in [10].

\section{Authors' contributions}

The main contributions of our work are as follows: (1) D-S theory is applied to increase the reliability of change detection. (2) The fuzzy exponent of FCM objection function is adaptively determined by conflict degree of evidence. All authors read and approved the final manuscript.

\section{Competing interests}

The authors declare that they have no competing interests.

\section{Author details}

${ }^{1}$ College of Computer and Information, Hohai University, No.8, Focheng West Road, Nanjing, China. ${ }^{2}$ Spatial Information Research Center, Changjiang Science Institute, No. 23, Huangpu Road, Wuhan, China.

Received: 22 April 2016 Accepted: 5 September 2016

Published online: 13 September 2016

References

1. D Lu, P Mausel, E Brondízio, E Moran, Change detection techniques. Int. J. Remote Sens. 25(12), 2365-2401 (2004)

2. RJ Radke, S Andra, O Al-Kofahi, B Roysam, Image change detection algorithms: a systematic survey. IEEE Trans. Image Process. 14(3), 294-307 (2005)

3. L Bruzzone, DF Prieto, Automatic analysis of the difference image for unsupervised change detection. IEEE Trans. Geosci. Remote Sens. 38(3), 1171-1182 (2000)

4. F Bovolo, L Bruzzone, M Marconcini, A novel approach to unsupervised change detection based on a semisupervised SVM and a similarity measure. IEEE Trans. Geosci. Remote Sens. 46(7), 2070-2082 (2008)

5. F Bovolo, G Camps-Valls, L Bruzzone, A support vector domain method for change detection in multitemporal images. Pattern Recogn. Lett. 31, 1148-1154 (2010)

6. Y Bazi, F Melgani, HD Al-Sharari, Unsupervised change detection in multispectral remotely sensed imagery with level set methods. IEEE Trans. Geosci. Remote Sens. 48(8), 3178-3187 (2010)

7. TCelik, K-K Ma, Unsupervised change detection for satellite images using dual-tree complex wavelet transform. IEEE Trans. Geosci. Remote Sens. 48(3), 1199-1210 (2010)

8. M Gong, Z Zhou, J Ma, Change detection in synthetic aperture radar images based on image fusion and fuzzy clustering. IEEE Trans. Image Process. 21(4), 2141-2151 (2012)

9. M Gong, Y Li, L Jiao, M Jia, L Su, Sar change detection based on intensity and texture changes. ISPRS J. Photogramm. Remote. Sens. 93, 123-125 (2014)

10. M Gong, L Su, M Jia, W Chen, Fuzzy clustering with a modified MRF energy function for change detection in synthetic aperture radar images. IEEE Trans. Fuzzy Syst. 22(1), 98-109 (2014)

11. M Gong, J Zhao, J Liu, Q Miao, L Jiao, Change detection in synthetic aperture radar images based on deep neural networks. IEEE Trans. Neural Netw. Learn. Syst. 27(1), 125-138 (2016)

12. $H$ Li, M Gong, Q Wang, J Liu, L Su, A multiobjective fuzzy clustering method for change detection in SAR images. Appl. Soft Comput. 46, 767-777 (2016)

13. PF He, WZ Shi, H Zhang, M Hao, A novel dynamic threshold method for unsupervised change detection from remotely sensed images. Remote Sens. Lett. 5(4), 396-403 (2014)

14. H Zhuang, K Deng, H Fan, M Yu, Strategies combining spectral angle mapper and change vector analysis to unsupervised change detection in multispectral images. IEEE Geosci. Remote Sens. Lett. 13(5), 681-685 (2016)

15. G Camps-Valls, L Gomez-Chova, J Munoz-Mari, JL Rojo-Alvarez, M Martinez-Ramon, Kernel-based framework for multitemporal and multisource remote sensing data classification and change detection. IEEE Trans. Geosci. Remote Sens. 46(6), 1822-1835 (2008)

16. M Volpi, D Tuia, F Bovolo, M Kanevski, L Bruzzone, Supervised change detection in VHR images using contextual information and support vector machines. Int. J. Appl. Earth Obs. Geoinformation. 20, 77-85 (2013)

17. T Celik, Unsupervised change detection in satellite images using principa component analysis and k-means clustering. IEEE Geosci. Remote Sens. Lett. 6(4), 772-776 (2009)

18. AA Nielsen, The regularized iteratively reweighted MAD method for change detection in multi- and hyperspectral data. IEEE Trans. Image Process. 16(2), 463-478 (2007)

19. MK Ridd, JJ Liu, A comparison of four algorithms for change detection in an urban environment. Remote. Sens. Environ. 63, 95-100 (1998)

20. AY Shi, DQ Huynh, FC Huang, SH Shen, WP Lu, ZL Ma, Unsupervised change detection based on robust chi-squared transform for bitemporal remotely sensed images. Int. J. Remote Sens. 35(21), 7555-7566 (2014) 
21. A Ghosh, NS Mishra, S Ghosh, Fuzzy clustering algorithms for unsupervised change detection in remote sensing images. Inf. Sci. 181(4), 699-715 (2011)

22. NS Mishra, S Ghosh, A Ghosh, Fuzzy clustering algorithms incorporating local information for change detection in remotely sensed images. Appl. Soft Comput. 12, 2683-2692 (2012)

23. BN Subudhi, F Bovolo, A Ghosh, L Bruzzone, Spatio-contextual fuzzy clustering with Markov random field model for change detection in remotely sensed images. Opt. Laser Technol. 57(0), 284-292 (2014)

24. P Shao, W Shi, P He, M Hao, X Zhang, Novel approach to unsupervised change detection based on a robust semi-supervised FCM clustering algorithm. Remote Sens. 8(264), 1-25 (2016)

25. P Du, S Liu, P Gamba, KTan, J Xia, Fusion of difference images for change detection over urban areas. IEEE J. Sel. Top. Appl. Earth Obs. Remote Sens. 5(4), 1076-1086 (2012)

26. SL Hegarat-Mascle, R Seltz, L Hubert-Moy, S Corgne, N Stach, Performance of change detection using remotely sensed data and evidential fusion: comparison of three cases of application. Int. J. Remote Sens. 27(16), 3515-3532 (2006)

27. AP Dempster, A generalization of Bayesian inference. J. R. Stat. Soc. Ser. B Methodol. 30(2), 205-247 (1968)

28. G Shafer, A Mathematical Theory of Evidence. (Princeton University Press, New Jersey, USA, 1976)

29. JC Dunn, A graph theoretic analysis of pattern classification via tamura's fuzzy relation. IEEE Trans. Syst. Man Cybern. SMC-4(3), 310-313 (1974)

30. JC Bezdek, Patter Recognition with Fuzzy Objective Function Algorithms. (Kluwer Academic Publishers, New York, 1981)

31. F Bovolo, G Camps-Valls, L Bruzzone, A support vector domain method for change detection in multitemporal images. Pattern Recogn. Lett. 31(10), 1148-1154 (2010). doi:10.1016/j.patrec.2009.07.002

32. N Otsu, A threshold selection method from gray-level histograms. IEEE Trans. Syst. Man Cybern. 9(1), 62-66 (1979)

33. Y Zhu, L Bentabet, O Dupuis, V Kaftandjian, D Dabot, M Rombaut, Automatic determination of mass functions in Dempster-Shafer theory using fuzzy c-means and spatial neighborhood information for image segmentation. Opt. Eng. 41(4), 760-770 (2002)

\section{Submit your manuscript to a SpringerOpen ${ }^{\circ}$ journal and benefit from:}

- Convenient online submission

- Rigorous peer review

- Immediate publication on acceptance

- Open access: articles freely available online

- High visibility within the field

- Retaining the copyright to your article

Submit your next manuscript at $\gg$ springeropen.com 